\title{
The effects of Pinoresinol diglucoside on the differentiation and bone resorption of osteoclast RAW264.7
}

\author{
Zhefeng JIN ${ }^{1}$, Hongtao $\mathrm{LI}^{2}$, Fangshan $\mathrm{BI}^{3}$, Hongmei CAO ${ }^{4 *}$ (D)
}

\begin{abstract}
The paper aimed to investigate the effect of Pinoresinol diglucoside on the differentiation and bone resorption of osteoclast RAW264.7, and to evaluate the effect of Pinoresinol diglucoside on osteoclasts.

In order to study the effect of Pinoresinol diglucoside on osteoclast differentiation, RANKL was used to induce RAW264.7 cells to differentiate into osteoclasts, and different concentrations of Pinoresinol diglucoside was added to intervene. CCK-8 method was applied to detect cell viability. TRAP staining was employed to observe cell morphology. Annexin V/PI flow cytometry was used to detect cell apoptosis. Phalloidin was used to detect F-actin formation of osteoclasts and to observe the effect on bone resorption. WB was employed to detect the effects of differentiation-related proteins and RANKL/RANK signaling pathways, and immunofluorescence detection technology was used to measure the distribution and nuclear translocation of p65. Pinoresinol diglucoside can effectively inhibit RANKL-induced osteoclast differentiation, especially in the early stage. The drug can inhibit the formation of F-actin of osteoclasts and inhibit bone resorption. Through inhibiting the ubiquitination and degradation of the homologous phosphatase tensin (PTEN), the drug up-regulated the viability of PTEN. The up-regulated PTEN viability then inhibited the NF- $\kappa B$ and AKT signaling pathways, resulting in a decrease in the expressions of nuclear factor $c 1$ (NFATc1) in activated T cells. Pinoresinol diglucoside effectively inhibited the formation of F-actin and bone resorption in mature osteoclasts. The mechanism is through inhibiting the expression levels of osteoclast-related proteins NFATc1, c-Fos, CSTK and TRAP and RNAKL/RANK signaling pathways, and also inhibiting the activation of NF- $\mathrm{KB}$ and AKT signaling pathways.
\end{abstract}

Keywords: Pinoresinol diglucoside; RAW264.7 cells; RNAKL/RANK; NF-кB; AKT.

Practical Application: Pinoresinol diglucoside on the Differentiation and Bone Resorption.

\section{Introduction}

Bone is a tissue that is constantly being renewed and remodeled. Osteoblasts synthesize bone matrix and osteoclasts absorb bone (Aspray \& Hill, 2019). Various small disturbances, such as the levels of inflammatory factors, growth factors and hormones, may cause the delicate balance between osteogenesis and osteoclastogenesis to be broken, leading to bone-related diseases. There are many diseases that are related to the balance breakdown between bone formation and bone resorption, such as osteoporosis, Paget's disease, rheumatoid arthritis, periodontal disease, and loosening around the prosthesis(Yu \& Xia, 2019). Excessive activation of osteoclasts is the main cause of the occurrence of these diseases(Anthamatten \& Parish, 2019; Tanaka, 2019)Osteoclasts are the only cells that perform bone resorption in the human body. They are derived from hematopoietic stem cell lines and are a kind of multinucleated giant cell fused under the stimulation of the macrophage colony stimulating factor (M-CSF) and nuclear factor- $\mathrm{KB}$ receptors factor ligand (RANKL) of monocytes/macrophages(Khosla \& Hofbauer,2 017; Jia et al., 2018).

In recent years, the pharmacodynamic and pharmacological research of natural compounds has become a research hotspot in various fields. Pinoresinol diglucoside is a lignan compound extracted from Eucommia. As a traditional Chinese medicine, Eucommia has been used for thousands of years. In many clinical trials, Pinoresinol diglucoside has been shown to have a variety of pharmacological activities, including anti-tumor, anti-inflammatory, anti-diabetic, antioxidant, cardioprotective and neuroprotective effects. But so far, the effect of Pinoresinol diglucoside on osteoclasts and osteoclast-related diseases has not been fully evaluated. In our research, we will focus on the effects of Pinoresinol diglucoside on RANKL-induced osteoclast differentiation, apoptosis, osteoclast-related protein expression, and bone resorption. 


\section{Methods}

\subsection{CCK-8 method to detect the effect of different concentrations of Pinoresinol diglucoside on the viability of RAW264.7 cells}

RAW264.7 cells were obtained and added with osteoclast differentiation media of different concentrations of Pinoresinol diglucoside. The Pinoresinol diglucoside concentrations are set at $0,10,20,40,80,160,320,640$, and $1280 \mu \mathrm{M}$. A multifunctional microplate reader was used to measure absorbance at $450 \mathrm{~nm}$ wavelength, and the reference wavelength is $650 \mathrm{~nm}$. With the DMSO group as the control group, the toxicity of Pinoresinol diglucoside at different concentrations to RAW264.7 cells was observed according to the measurement results.

\subsection{TRAP staining to observe the effect of different concentrations of Pinoresinol diglucoside on the process of RAW264.7 inducing osteoclast differentiation}

RAW264.7 cells were prepared, added with Pinoresinol diglucoside at concentrations of $0,20,40$, and $80 \mu \mathrm{M}$, fixed with $4 \%$ paraformaldehyde, then stained with TRAP and observed under a light microscope.

\subsection{Annexin V/PI flow cytometry detection of apoptosis}

RAW264.7 cells were prepared and induced to differentiate into osteoclasts for 3 days. During this period, 0, 20, 40, and 80 $\mu \mathrm{M}$ Pinoresinol diglucoside were added to observe the effect of the drug on osteoclast apoptosis.

\subsection{Western blotting to detect the effect of the drug on various proteins.}

RAW264.7 cells were obtained and induced to differentiate into osteoclasts for 3 days. During this period, 0, 20, 40, and 80 $\mu \mathrm{M}$ Pinoresinol diglucoside is added. Following this, protein sample was prepared. SDS-PAGE gel electrophoresis and membrane transference were conducted. The cells were added with apoptosis-related proteins and corresponding antibodies of the RANKL/RANK signaling pathway for incubation and subsequent ECL development. Image J software was used for quantitative analysis.

\subsection{Osteoclast F-actin ring detection}

RAW264.7 cells were obtained and induced to differentiate into osteoclasts for 3 days. During this period, 20, 40, and 80 $\mu \mathrm{M}$ Pinoresinol diglucoside were added. Phalloidin was added to PBS buffer and mixed evenly to cover the bovine bone slices. DAPI counterstains the nuclei. The bovine bone slices were taken out and mounted with anti-fluorescence quenching mounting tablets, and observed under a fluorescence microscope. The number and morphology of osteoclast F-actin were studied. Image J software was used for statistical analysis.

\subsection{Cellular immunofluorescence experiment to observe the distribution of p65 in cells and nucleus}

RAW264.7 cells were obtained and added with $80 \mu \mathrm{M}$ Pinoresinol diglucoside. The cells were observed under a fluorescent microscope using immunofluorescence method. The distribution of p65 in the cells and the nucleus were studied, and pictures were taken for analysis.

\section{Result}

\subsection{The effect of Pinoresinol diglucoside on the viability of mouse RAW264.7 cells}

In order to analyze the effect of different concentrations of Pinoresinol diglucoside on the viability of RAW264.7 cells, RANKL was used to induce RAW264.7 cells to differentiate into osteoclasts for 5 days. During the differentiation process, different experimental groups were added with different concentrations of Pinoresinol diglucoside respectively. CCK- 8 kit was used to test cell viability on the 5 th day. The results showed that, Pinoresinol diglucoside of all the concentrations did not affect the viability of RAW264.7 cells, proving that Pinoresinol diglucoside at a concentration of $1280 \mu \mathrm{M}$ and below has no obvious cytotoxicity to RAW264.7, as shown in Figure 1.

\subsection{The effect of Pinoresinol diglucoside on the differentiation of RAW264.7 cells into osteoclasts}

In the $0 \mu \mathrm{M}$ group, RAW264.7 cells can be seen to differentiate into larger purple multinucleated cells with multiple nuclei, and in large numbers. In contrast, under the conditions of 20, 40, 80 $\mu \mathrm{M}$ Pinoresinol diglucoside incubation, the volume of osteoclasts was significantly reduced, and the number of multinucleated cells was also reduced, suggesting that Pinoresinol diglucoside can inhibit the process of RANKL inducing RAW264.7 cells differentiate into osteoclasts. As shown in Figure 2.

\subsection{The effect of Pinoresinol diglucoside on the apoptosis of RAW264.7 cells}

Apoptotic cells were detected by flow cytometry. The results showed that, compared with the $0 \mu \mathrm{M}$ group, Pinoresinol diglucoside did not cause osteoclast apoptosis, and there was

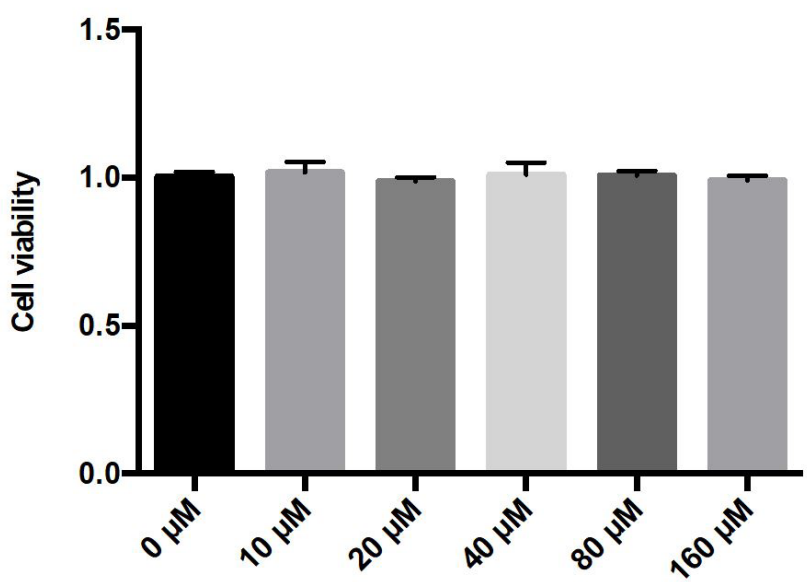

Figure 1. The effect of Pinoresinol diglucoside on the viability of RAW264.7 cells. 
no significant difference in the proportion of apoptotic cells between the groups, as shown in Figure 3.

\subsection{The effect of Pinoresinol diglucoside on the F-actin ring of osteoclasts}

Rhodamine-labeled phalloidin was used to stain F-actin. Fluorescence microscopy showed that in the $0 \mu \mathrm{M}$ group, osteoclasts formed a large and complete F-actin ring structure. In the Pinoresinol diglucoside treatment group, the F-actin ring structure was significantly damaged, especially at the concentration of $80 \mu \mathrm{M}$, in which no complete F-actin ring structure was observed. Instead, there were small scattered actin particles, as shown in Figure 4.

\subsection{The effect of Pinoresinol diglucoside on bone resorption of osteoclasts}

In the $0 \mu \mathrm{M}$ group, a large number of bone resorption lacunas can be seen on the surface of the bone slices. However, in the Pinoresinol diglucoside treatment group, bone resorption lacunas were significantly reduced, especially in the $80 \mu \mathrm{M}$ group almost no Bone resorption lacuna was observed, see Figure 5.

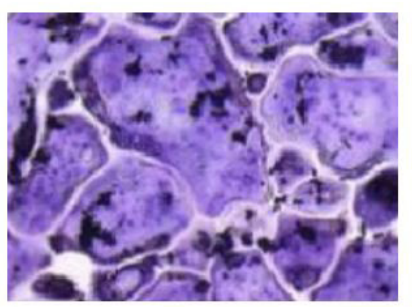

$0 \mu \mathrm{M}$

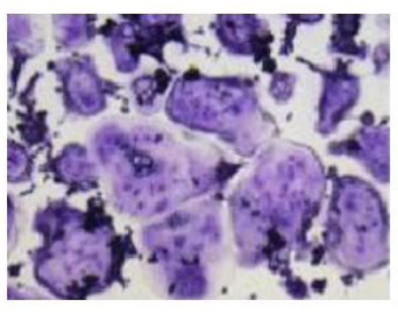

$20 \mu \mathrm{M}$
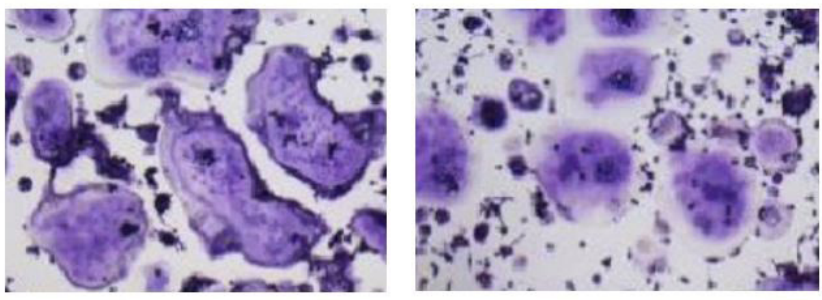

$40 \mu \mathrm{M}$

Figure 2. The effect of Pinoresinol diglucoside on the differentiation of RAW264.7 cells into osteoclasts.

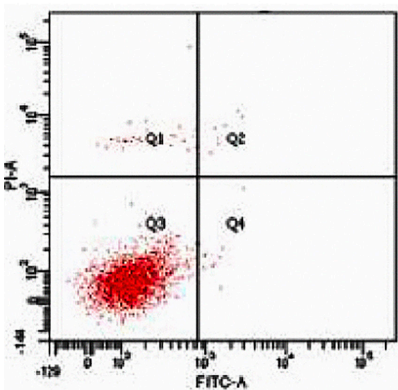

$0 \mu \mathrm{M}$

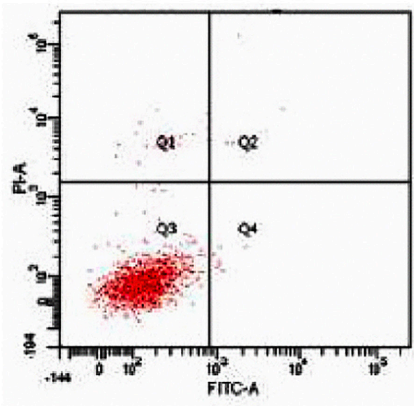

$20 \mu \mathrm{M}$

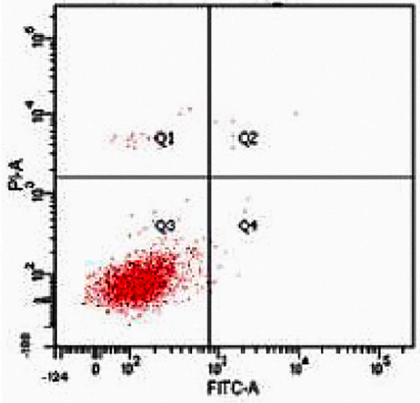

$40 \mu \mathrm{M}$

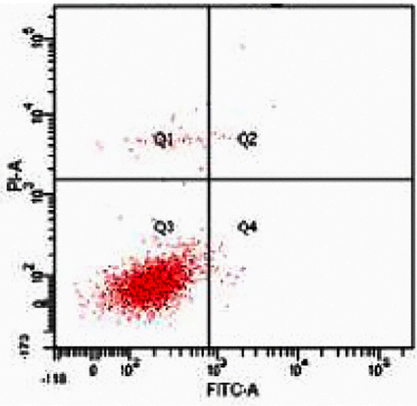

$80 \mu \mathrm{M}$

Figure 3. The effect of Pinoresinol diglucoside on the apoptosis of RAW264.7 cells.

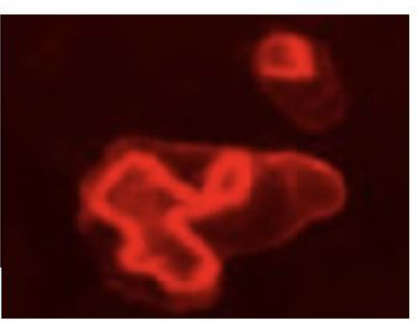

$0 \mu \mathrm{M}$

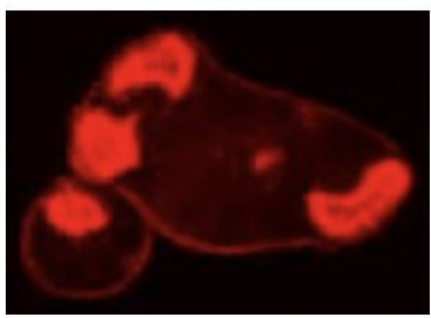

$20 \mu \mathrm{M}$

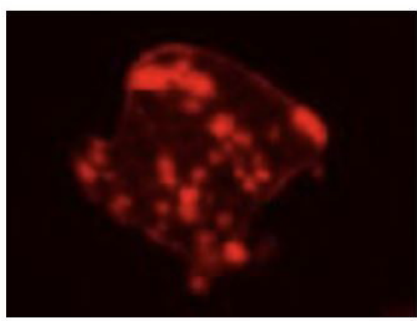

$40 \mu \mathrm{M}$

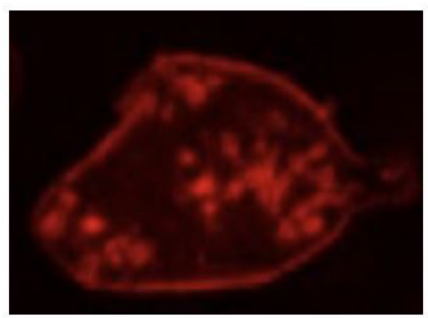

$80 \mu \mathrm{M}$

Figure 4. The effect of Pinoresinol diglucoside on the F-actin ring of osteoclasts. 


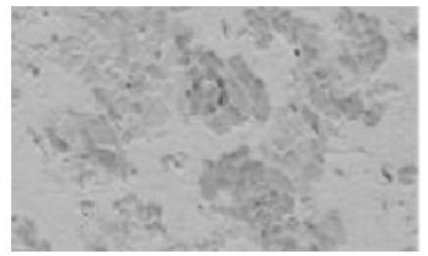

$0 \mu \mathrm{M}$

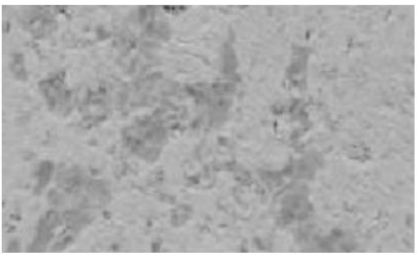

$20 \mu \mathrm{M}$

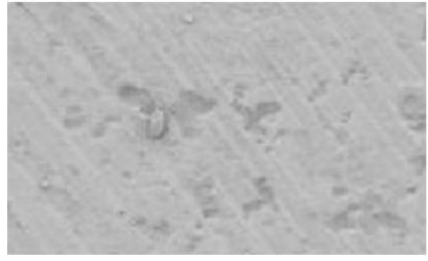

$40 \mu \mathrm{M}$

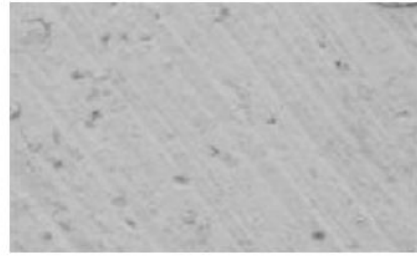

$80 \mu \mathrm{M}$

Figure 5. The effect of Pinoresinol diglucoside on the bone resorption of osteoclasts.

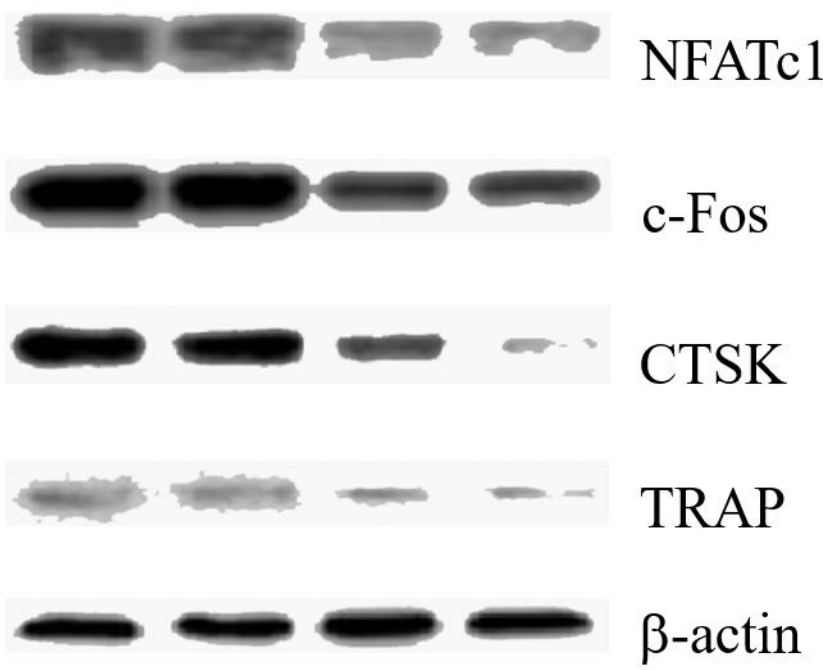

Figure 6. The effect of Pinoresinol diglucoside on NFATc1, c-Fos, CTSK and TRAP proteins.

\subsection{The effect of Pinoresinol diglucoside on osteoclast differentiation-related proteins}

The results showed that, after Pinoresinol diglucoside treatment, the expressions of NFATc1, c-Fos, CTSK and TRAP proteins were significantly inhibited, suggesting that the drug inhibited osteoclast differentiation and maturation, as shown in Figure 6.

Due to the effect of Pinoresinol diglucoside on RANKL/ RANK signaling pathway, the drug significantly inhibited the phosphorylation of I $\mathrm{K} \mathrm{B} \alpha$ and $\mathrm{p} 65$, and inhibited the degradation of I $\kappa \mathrm{B} \alpha$, proving its inhibitory effect on NF- $\mathrm{KB}$ signals. At the same time, Pinoresinol diglucoside inhibited the phosphorylation of AKT and GSK-3 $\beta$, indicating that the drug may inhibit osteoclasts through the AKT-GSK-3 $\beta$-NFATc1 signaling axis, as shown in Figure 7.

\subsection{The effect of Pinoresinol diglucoside on the nuclear translocation of p65 induced by RANKL}

Fluorescence microscopy showed that p65 was evenly distributed throughout the macrophages in the absence of RANKL stimulation. After RANKL stimulation for 20 minutes,

\section{$0 \mu \mathrm{M} 20 \mu \mathrm{M} 40 \mu \mathrm{M} 80 \mu \mathrm{M}$}
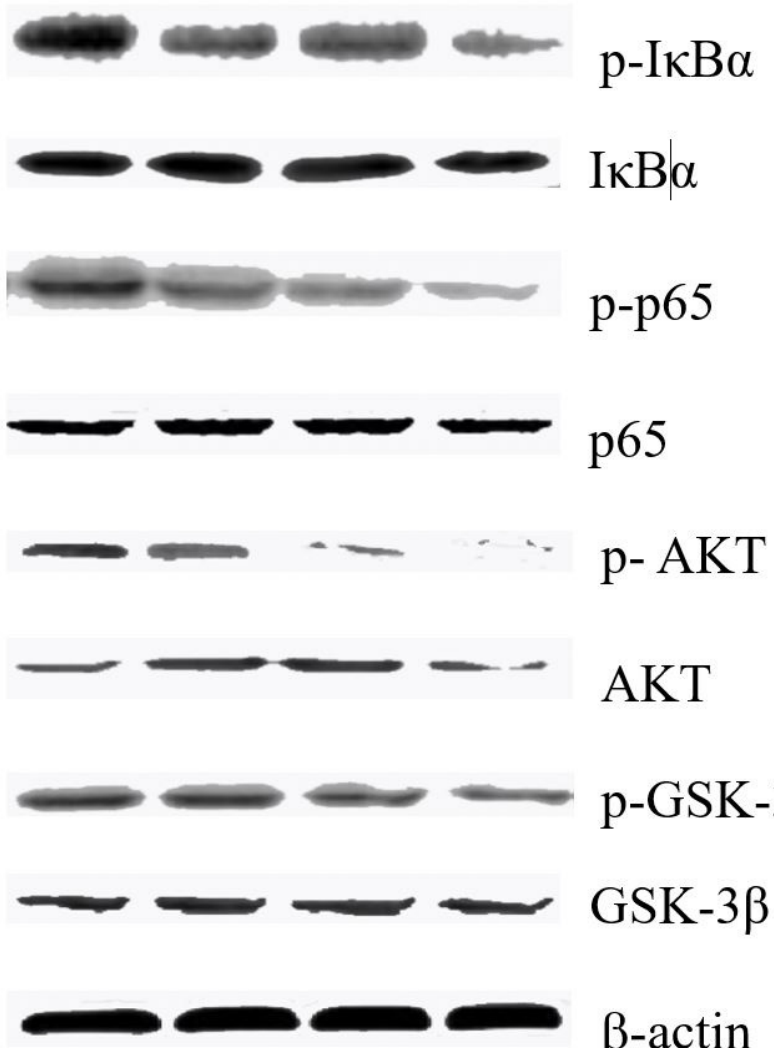

p-GSK-3 $\beta$

GSK-3 $\beta$

$\beta$-actin

Figure 7. The effect of Pinoresinol diglucoside on RANKL/RANK signaling pathway.

most of p65 was concentrated in the nucleus, while p65 in the cytoplasm was relatively reduced. In the Pinoresinol diglucoside treatment group, p65 was evenly distributed in the cells, and most of the p65 in the cells was still distributed in the syrup, proving that Pinoresinol diglucoside can effectively inhibit the nuclear translocation of p65 induced by RANKL, as shown in Figure 8 .

\section{Discussion}

RANKL/RANK signaling pathway plays an important role in the process of osteoclast survival, differentiation and bone resorption(Kim B,et al.,2018). When RANKL binds to its receptor 


\section{$0 \mu \mathrm{M}$}
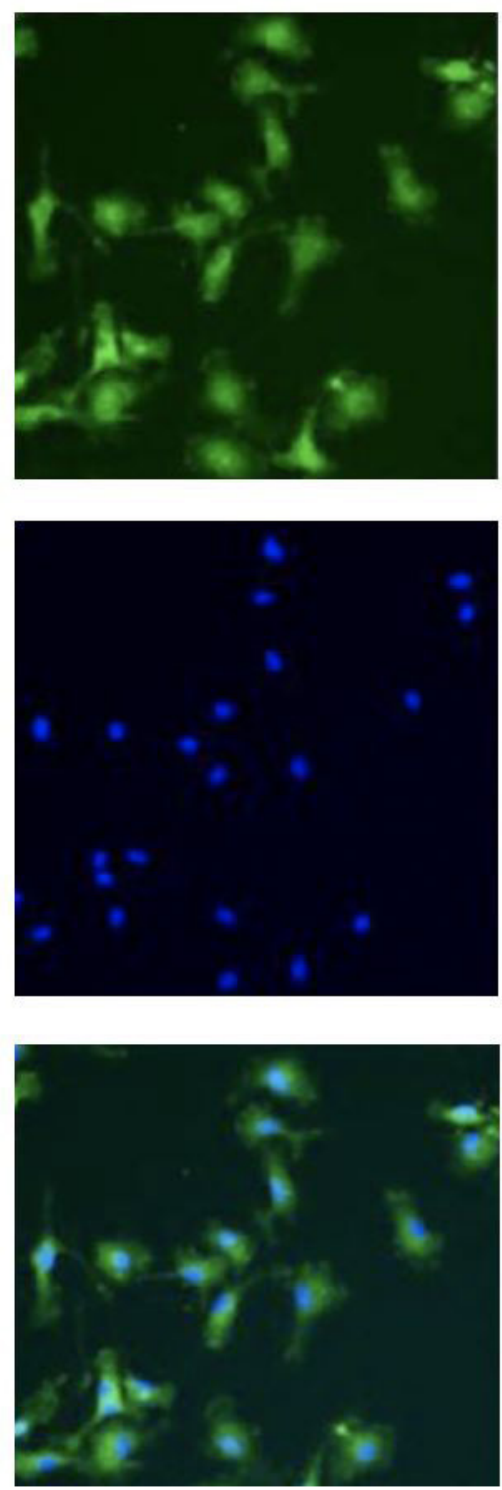

50ng/ml RANKL

\section{$80 \mu \mathrm{M}+50 \mathrm{ng} / \mathrm{ml} \mathrm{RANKL}$}
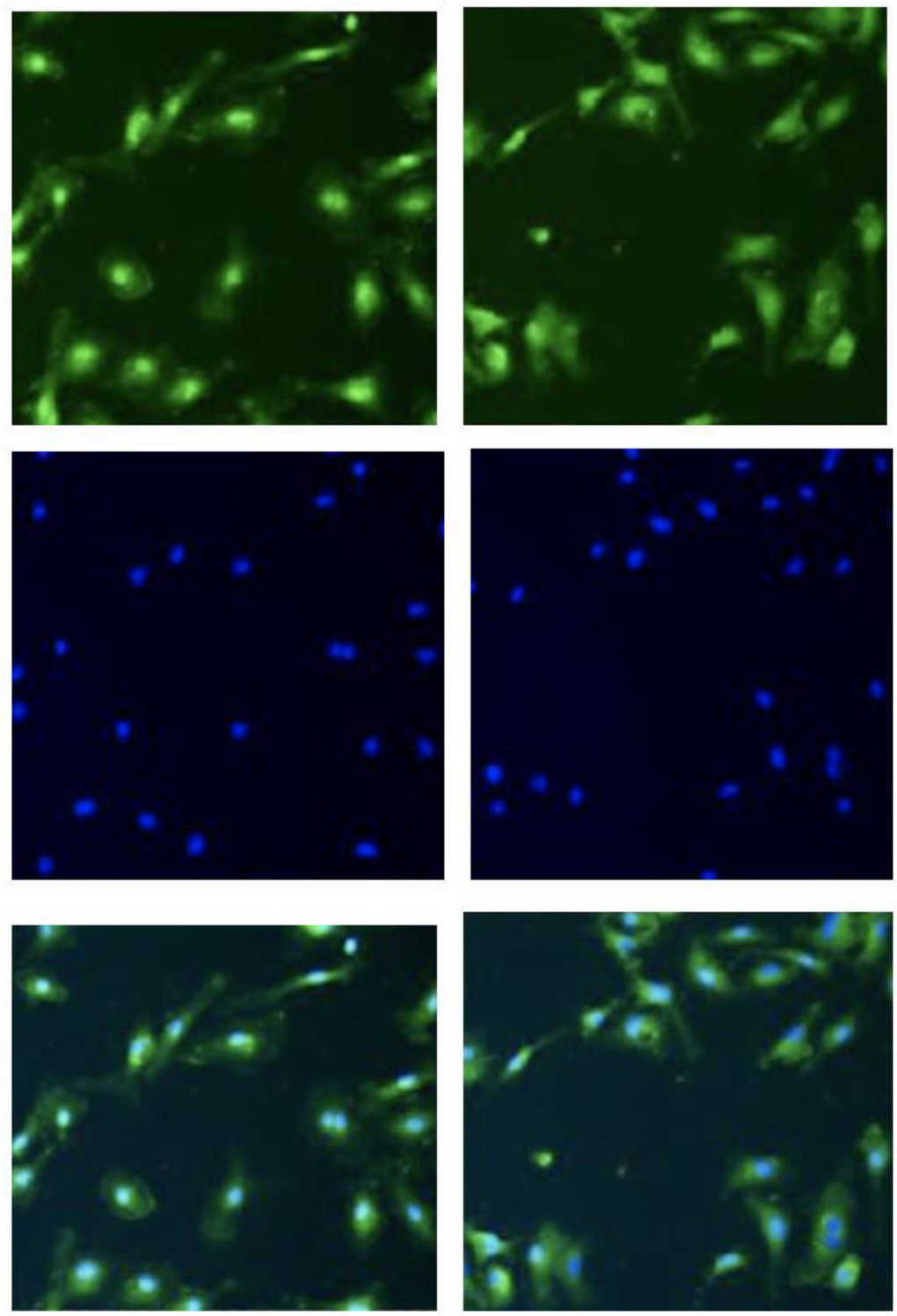

Figure 8. The effect of Pinoresinol diglucoside on the nuclear translocation of p65 induced by RANKL.

RANK, it will induce the activation of five intracellular signaling pathways, including the NF- $\kappa$ B signal, AKT signal and MAPK signal (MAPK includes ERK, JNK and p38 signals)(Xu et al., 2019). In the RANKL/RANK signal, the activation of the NF- $\kappa B$ signal starts from the RANK/TRAF6/TAK1 complex, which can induce the phosphorylation of TAK1, activate its kinase activity, and then phosphorylate I $\kappa \mathrm{B}$ kinase (IKK), thereby initiating $\mathrm{NF}-\kappa \mathrm{B}$ signaling. In the resting state, NF- $\kappa \mathrm{B}$ and I $\kappa \mathrm{B}$ combine to exist in the cytoplasm and are in an inactive state. Under the stimulation of RANKL, the activated IKK catalyzes the phosphorylation of $\mathrm{I} \kappa \mathrm{B}$ and promotes degradation, dissociating the NF- $\kappa \mathrm{B} / \mathrm{I} \kappa \mathrm{B}$ complex, thereby releasing NF- $\kappa \mathrm{B}$ p 65 . NF- $\kappa \mathrm{B}$ p65 has a nuclear localization sequence, which can initiate the transcription of downstream related genes after being guided into the nucleus (Choi 2019; Liu et al., 2017) In this study, we found that Pinoresinol diglucoside can inhibit the phosphorylation and degradation of I $\kappa \mathrm{B} a$ induced by RANKL, thereby inhibiting the phosphorylation of NF- $\kappa \mathrm{B}$ p65. In addition, through cellular immunofluorescence, we also confirmed that Pinoresinol diglucoside can inhibit the nuclear translocation of NF- $\kappa \mathrm{B}$ p65.

The AKT/GSK3 $\beta /$ NFATc1 signal is also a very important part of the RANKL/RANK signal. Under RANKL stimulation, TRAF6 can activate PI3K/AKT and promote the phosphorylation of both signals. Subsequently, the activated AKT can phosphorylate and activate GSK3 $\beta$. The activated GSK3 $\beta$ can promote the nuclear translocation of NFATc1, thereby promoting osteoclast differentiation(Shalev \& Elson, 2019). The results of the experiment showed that Pinoresinol diglucoside can inhibit AKT and GSK3 $\beta$, 
indicating that the inhibitory effect of catalpol on RANKL/ RANK signaling involves multiple signaling pathways. NFATc1 is a member of the NFAT family of transcription factors. Its activation and increased expression are key events in osteoclast differentiation. Embryonic stem cells knocked out by NFATc1 cannot differentiate into osteoclasts under the stimulation of RANKL(Meng et al., 2019). Both NF- $\mathrm{kB}$ and AKT signals regulate osteoclast differentiation related to NFATc1(Ye et al., 2018; Wu et al., 2017). After entering the nucleus, NF- $\mathrm{KB}$ p65 can bind to the promoter of NFATc1 to initiate early transcription of NFATc1 (Khalifeh-Soltani et al., 2018). NFATc1 is a very critical transcription factor in the process of osteoclast differentiation. It can regulate the expressions of a series of osteoclast-related genes. Our research found that Pinoresinol diglucoside can inhibit the expression level of NFATc1 protein, which may be related to its inhibition of the NF- $\kappa \mathrm{B}$ and AKT signaling pathways. At the same time, Pinoresinol diglucoside also inhibited the expression levels of CTSK and TRAP proteins in NFATc1 downstream.

\section{Conclusion}

Our research found that Pinoresinol diglucoside effectively inhibited the formation of F-actin and bone resorption in mature osteoclasts. The mechanism was through inhibiting the expression levels of osteoclast-related proteins NFATc1, c-Fos, CSTK and TRAP, as well as the activation of NF- $\mathrm{KB}$ and AKT signaling pathways in RNAKL/RANK.

\section{Reference}

Anthamatten, A., \& Parish, A. (2019). Clinical update on osteoporosis. Journal of Midwifery \& Women's Health, 64(3), 265-275. http://dx.doi. org/10.1111/jmwh.12954. PMid:30869832.

Aspray, T. J., \& Hill, T. R. (2019). Osteoporosis and the Ageing Skeleton. Sub-Cellular Biochemistry, 91, 453-476. http://dx.doi.org/10.1007/978981-13-3681-2_16. PMid:30888662.

Choi, Y. H. (2019). Catalpol attenuates lipopolysaccharide-induced inflammatory responses in BV2 microglia through inhibiting the TLR4-mediated NF-kappaB pathway. General Physiology and Biophysics, 38(2), 111-122. http://dx.doi.org/10.4149/gpb-2018044. PMid:30806632.

Jia, Y., Miao, Y., Yue, M., Shu, M., Wei, Z., \& Dai, Y. (2018). Tetrandrine attenuates the bone erosion in collagen-induced arthritis rats by inhibiting osteoclastogenesis via spleen tyrosine kinase. The FASEB Journal, 32(6), 3398-3410. http://dx.doi.org/10.1096/fj.201701148RR. PMid:29401630.
Khalifeh-Soltani, A., Gupta, D., Ha, A., Podolsky, M. J., Datta, R., \& Atabai, K. (2018). The Mfge8-alpha8beta1-PTEN pathway regulates airway smooth muscle contraction in allergic inflammation. The FASEB Journal, 32(11), 5927-5936. PMid:29763381.

Khosla, S., \& Hofbauer, L. C. (2017). Osteoporosis treatment: recent developments and ongoing challenges. The Lancet. Diabetes \& Endocrinology, 5(11), 898-907. http://dx.doi.org/10.1016/S22138587(17)30188-2. PMid:28689769.

Kim B., Lee K. Y., \& Park B. (2018). Icariin abrogates osteoclast formation through the regulation of the RANKL-mediated TRAF6/NF-kappaB/ ERK signaling pathway in Raw264.7 cells. Phytomedicine, 51, 181-190.

Liu, L., Gao, H., Wang, H., Zhang, Y., Xu, W., Lin, S., Wang, H., Wu, Q., \& Guo, J. (2017). Catalpol promotes cellular apoptosis in human HCT116 colorectal cancer cells via microRNA-200 and the downregulation of PI3K-Akt signaling pathway. Oncology Letters, 14(3), 3741-3747. http://dx.doi.org/10.3892/ol.2017.6580. PMid:28927141.

Meng, J., Zhou, C., Zhang, W., Wang, W., He, B., Hu, B., Jiang, G., Wang, Y., Hong, J., Li, S., He, J., Yan, S., \& Yan, W. (2019). Stachydrine prevents LPS-induced bone loss by inhibiting osteoclastogenesis via NF-kappaB and Akt signaling. Journal of Cellular and Molecular Medicine, 23(10), 6730-6743. PMid:31328430.

Shalev, M., \& Elson, A. (2019). The roles of protein tyrosine phosphatases in bone-resorbing osteoclasts. Biochimica et Biophysica Acta. Molecular Cell Research, 1866(1), 114-123. http://dx.doi.org/10.1016/j. bbamcr.2018.07.005. PMid:30026076.

Tanaka, S. (2019). Molecular understanding of pharmacological treatment of osteoporosis. EFORT Open Reviews, 4(4), 158-164. http://dx.doi. org/10.1302/2058-5241.4.180018. PMid:31057953.

Wu, M., Chen, W., Lu, Y., Zhu, G., Hao, L., \& Li, Y. P. (2017). Galpha13 negatively controls osteoclastogenesis through inhibition of the AktGSK3beta-NFATc1 signaling pathway. Nature Communications, 8(1), 13700. http://dx.doi.org/10.1038/ncomms13700. PMid:28102206.

Xu, Q., Chen, G., Liu, X., Dai, M., \& Zhang, B. (2019). Icariin inhibits RANKL-induced osteoclastogenesis via modulation of the NF-kappaB and MAPK signaling pathways. Biochemical and Biophysical Research Communications, 508(3), 902-906. http://dx.doi.org/10.1016/j. bbrc.2018.11.201. PMid:30538045.

Ye, L., Lou, F., Yu, F., Zhang, D., Wang, C., Wu, F., Li, X., Ping, Y., Yang, X., Yang, J., Chen, D., Gao, B., Huang, D., \& Liu, P. (2018). NUMB maintains bone mass by promoting degradation of PTEN and GLI1 via ubiquitination in osteoblasts. Bone Research, 6(1), 32. http:// dx.doi.org/10.1038/s41413-018-0030-y. PMid:30455992.

Yu, F., \& Xia, W. (2019). The epidemiology of osteoporosis, associated fragility fractures and management gap in China. Archives of Osteoporosis, 14(1), 32. http://dx.doi.org/10.1007/s11657-0180549-y. PMid:30848398. 